\title{
Elaborate hallucinations, but is it a psychotic disorder?
}

John Wang, MD, Dina Patel, MD, and Dimitry Francois, MD

\section{How would you handle this case?}

Answer the challenge questions at MDedge.com/ psychiatry and see how your colleagues responded

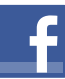

Discuss this article at www.facebook.com/ MDedgePsychiatry

Mr. B, age 93, has recurrent visual, auditory, and tactile hallucinations involving a man named 'Harry', but no other psychotic symptoms. What diagnoses should you consider?

\section{CASE Visual, auditory, and tactile hallucinations}

Mr. B, age 93, is brought to the emergency department by his son after experiencing hallucinations where he reportedly saw and heard individuals in his home. In frustration, Mr. B wielded a knife because he "wanted them to go away."

Mr. B and his son report that the hallucinations had begun 2 years ago, without prior trauma, medication changes, changes in social situation, or other apparent precipitating events. The hallucinations "come and go," without preceding symptoms, but have recurring content involving a friendly man named "Harry," people coming out of the television, 2 children playing, and water covering the floor. Mr. B acknowledges these are hallucinations and had not felt threatened by them until recently, when he wielded the knife. He often tries to talk to them, but they do not reply.

Mr. B also reports intermittent auditory hallucinations including voices at home (non-command) and papers rustling. He also describes tactile hallucinations, where he says he can feel Harry and others prodding him, knocking things out of his hands, or splashing him with water.

Mr. B is admitted to the hospital because he is a danger to himself and others. While on the inpatient unit, Mr. B is pleasant with staff, and eats and sleeps normally; how- ever, he continues to have hallucinations of Harry. Mr. B reports seeing Harry in the hall, and says that Harry pulls out Mr. B's earpiece and steals his fork. Mr. B also reports hearing a sound "like a bee buzzing." Mr. B is started on risperidone, $1 \mathrm{mg}$ nightly, for a presumed psychotic disorder.

\section{HISTORY Independent and in good health}

Mr. B lives alone and is independent in his activities of daily living. He spends his days at home, often visited by his children, who bring him groceries and other necessities.

Mr. B takes no medications, and has no history of psychiatric treatment; psychotic, manic, or depressive episodes; posttraumatic stress disorder; obsessive-compulsive disorder; or recent emotional stress. His medical history includes chronic progressive hearing loss, which is managed with hearing aids; macular degeneration; and prior bilateral cataract surgeries.

Dr. Wang is a PGY-1 Psychiatry Resident, Department of Psychiatry, New York University School of Medicine, New York, New York. Dr. Patel is a Fellow, Department of Psychiatry, Weill Cornell Medicine, New York, New York. Dr. Francois is Assistant Professor, Department of Psychiatry, Weill Cornell Medicine, New York, New York.

Disclosures

The authors report no financial relationships with any companies whose products are mentioned in this article, or with manufacturers of competing products.

doi: 10.12788/cp.0091 


\section{EVALUATION Mental status exam and objective findings}

During his evaluation, Mr. B appears wellnourished, and wears glasses and hearing aids. During the interview, he is euthymic with appropriately reactive affect. He is talkative but redirectable, with a goal-directed thought process. Mr. B does not appear to be internally preoccupied. His hearing is impaired, and he often requires questions to be repeated loudly. He is oriented to person, place, and time. There are no signs of delusions, paranoia, thought blocking, thought broadcasting/insertion, or referential thinking. He denies depressed mood, anhedonia, fatigue, sleep changes, or manic symptoms. He denies the occurrence of auditory or visual hallucinations during the evaluation.

A neurologic exam shows impaired hearing bilaterally and impaired visual acuity. Even with glasses, both eyes have acuity only to finger counting. All other cranial nerves are normal, and Mr. B's strength, sensation, and cerebellar function are all intact, without rigidity, numbness, or tingling. His gait is steady without a walker, with symmetric arm swing and slight dragging of his feet. His vitals are stable, with normal orthostatic pressures.

Other objective data include a score of 24/30 on the Mini-Mental State Examination, notable for deficits in visuospatial orientation, attention, and calculation, with language and copying limited by poor vision. Mr. B scores 16/22 on the Montreal Cognitive Assessment (MoCA)-Blind (adapted version of MoCA), which is equivalent to a 22/30 on the MoCA, indicating some mild cognitive impairment; however, this modified test is still limited by his poor hearing. His serum and urine laboratory workup show no liver, kidney, metabolic, or electrolyte abnormalities, no sign of infection, negative urine drug screen, and normal B12 and thyroidstimulating hormone levels. He undergoes a brain MRI, which shows chronic microvascular ischemic change, without mass lesions, infarction, or other pathology.
What should be included in the differential diagnosis for Mr. B?
a) schizophrenia
b) Charles Bonnet syndrome (CBS)
c) Lewy Body dementia
d) B and $C$

\section{The authors' observations}

Given Mr. B's presentation, we ruled out a primary psychotic disorder. He had no psychiatric history, with organized thought, a reactive affect, and no delusions, paranoia, or other psychotic symptoms, all pointing against psychosis. His brain MRI showed no malignancy or other lesions. He had no substance use history to suggest intoxication/withdrawal. His intact attention and orientation did not suggest delirium, and his serum and urine studies were all negative. Although his blaming Harry for knocking things out of his hands could suggest confabulation, Mr. B had no other signs of Korsakoff syndrome, such as ataxia, general confusion, or malnourishment.

We also considered early dementia. There was suspicion for Lewy body dementia given Mr. B's prominent fluctuating visual hallucinations; however, he displayed no other signs of the disorder, such as parkinsonism, dysautonomia, or sensitivity to the antipsychotic (risperidone $1 \mathrm{mg}$ nightly) started on admission. The presence of 1 core feature of Lewy body dementia-visual hallucinations-indicated a possible, but not probable, diagnosis. Additionally, Mr. B did not have the characteristic features of other types of dementia, such as the stepwise progression of vascular dementia, the behavioral disinhibition of frontotemporal dementia, or the insidious forgetfulness, confusion, language problems, or paranoia that may appear in Alzheimer's disease. Remarkably, he had a relatively normal brain MRI for his age, given chronic microvascular ischemic changes, and cognitive testing that indicated only mild impairment further pointed against a dementia process.

\section{Clinical Point}

We suspected that Mr. B may have had Lewy body dementia, given his prominent fluctuating visual hallucinations 


\section{Clinical Point}

\section{Charles Bonnet syndrome has been} observed in several disorders that affect vision, including macular degeneration and glaucoma

\section{Visual hallucinations in Charles Bonnet syndrome}

\begin{tabular}{l:l} 
Characteristic & Feature \\
\hline Appearance & $\begin{array}{l}\text { Most often simple shapes, but may be complex (faces, people, } \\
\text { scenes) }\end{array}$ \\
\hline Movement & May be either static or move around \\
\hline Duration & Range from a few seconds to several minutes or continuous \\
\hline Timing & Range from rare, isolated episodes to several times daily \\
\hline Exacerbating factors & $\begin{array}{l}\text { Evening/night, sensory deprivation (dim lighting), social isolation, } \\
\text { white backgrounds }\end{array}$ \\
\hline Alleviating factors & Certain eye movements, turning on more light, social interaction \\
\hline Co-occurring hallucinations & Usually do not occur with auditory or other hallucinations \\
\hline Source: Reference 3 &
\end{tabular}

\section{Charles Bonnet syndrome}

Based on Mr. B's severe vision loss and history of ocular surgeries, we diagnosed him with CBS, described as visual hallucinations in the presence of impaired vision. Charles Bonnet syndrome has been observed in several disorders that affect vision, most commonly macular degeneration, diabetic retinopathy, and glaucoma, with an estimated prevalence of $11 \%$ to $39 \%$ in older patients with ocular disease. ${ }^{1,2}$ Visual hallucinations in CBS occur due to ocular disease, likely resulting from changes in afferent sensory input to visual cortical regions of the brain. Table $\mathbf{1}^{3}$ outlines the features of visual hallucinations in patients with CBS. The subsequent disinhibition and spontaneous firing of the visual association cortices leads to the "release hallucinations" of the syndrome. ${ }^{4}$ The disorder is thought to be significantly underdiagnosed-in a survey of patients with CBS, only $15 \%$ had reported their visual hallucinations to a physician. ${ }^{5}$

Mr. B's symptoms are atypical for CBS, but they fit the diagnosis when considering the entire clinical picture. While hallucinations in CBS are more often simple shapes, complex hallucinations including people and scenes have been noted in several instances. ${ }^{6}$

Similar to Mr. B's case, patients with CBS can have recurring figures in their hallucinations, and the images may even move across the visual field. ${ }^{1}$ Patients with CBS also frequently recognize that their hallucinations are not real, and may or may not be distressed by them. ${ }^{4}$ Patients with CBS often have hallucinations multiple times daily, lasting from a few seconds to many minutes, ${ }^{7}$ consistent with Mr. B's temporary symptoms.

Although auditory and tactile hallucinations are typically not included in CBS, they can also be explained by Mr. B's significant sensory impairment. Severe hearing impairment in geriatric adults has been associated with auditory hallucinations ${ }^{8}$; in 1 survey, half of these hallucinations consisted of voices. ${ }^{9}$ In contrast, tactile hallucinations are not described in sensory deprivation literature. However, in the context of Mr. B's severe comorbid hearing and vision loss, we propose that these hallucinations reflect his interpretation of sensory events around him, and their integration into his extensive hallucination framework. In other words, Harry poking him and causing him to drop things may be Mr. B's way of rationalizing events that he has trouble perceiving entirely, or his mild forgetfulness. Mr. B's social isolation is another factor that may worsen his sensory deprivation and contribute to his extensive hallucinations. ${ }^{10}$ Additionally, his mild cognitive deficits 
on testing with chronic microvascular changes on the MRI may suggest a mild vascular-related dementia process, which could also exacerbate his hallucinations. While classic CBS occurs without cognitive impairment, dementia can often co-occur with CBS.11

\section{TREATMENT No significant improvement with medications}

During his inpatient stay, Mr. B is treated with risperidone, $1 \mathrm{mg}$ nightly, and is also started on donepezil, $5 \mathrm{mg} / \mathrm{d}$, to treat a possible comorbid dementia. However, he continues to hallucinate without significant improvement.

\section{Which approach is most effective to treat Charles Bonnet syndrome? \\ a) antipsychotics \\ b) donepezil or another anti-dementia medication \\ c) reassurance and social engagement}

\section{Table 2}

Treatment options for Charles Bonnet syndrome

\begin{tabular}{|l|}
\hline Medications \\
\hline Carbamazepine \\
\hline Clonazepam \\
\hline Donepezil \\
\hline Escitalopram \\
\hline Gabapentin \\
\hline Olanzapine \\
\hline Risperidone \\
\hline Valproate \\
\hline Venlafaxine \\
\hline Behavioral interventions \\
\hline Improved lighting \\
\hline Blinking/eye movement training \\
\hline Psychoeducation and groups \\
\hline Reassurance \\
\hline Encouraging interpersonal contact \\
\hline Distraction with another activity \\
\hline Talking to the hallucination \\
\hline Walking toward the hallucination \\
\hline Vision support, such as glasses \\
\hline Source: References 8,11 \\
\hline
\end{tabular}

such as donepezil, antiepileptics (carbamazepine, valproate, gabapentin, and clonazepam), and certain antidepressants (escitalopram, venlafaxine) (Table $\left.2^{8,11}\right)^{3}$

\section{Addressing loneliness and social isolation}

With minimal definitive evidence for pharmacologic management, the most important intervention for treating CBS may be changing the patient's sensory environment. Specifically, loneliness and social isolation are major exacerbating factors of CBS, and many clinicians advocate for the consistent presence of a sympathetic professional. Reassurance that hallucinations are from ocular disease rather than a primary mental disorder may be extremely relieving for patients. ${ }^{11}$ A psychoeducation or support group may also be beneficial, not only for giving patients more social contact, but also

\section{Clinical Point}

Many patients with
CBS can have the
hallucinations remit
after the underlying
ocular etiology is
corrected

Many patients with Can have the corred 


\section{Clinical Point}

The most important intervention for treating CBS may be changing the patient's sensory environment

\section{Related Resources}

- Charles Bonnet Syndrome Foundation. http://www. charlesbonnetsyndrome.org

- Schultz G, Melzack R. The Charles Bonnet syndrome: 'phantom visual images'. Perception. 1991;20:809-825.

- Menon GJ, Rahman I, Menon SJ, et al. Complex visual hallucinations in the visually impaired: the Charles Bonnet syndrome. Surv Ophthalmol. 2003;48(1):58-72.

\section{Drug Brand Names}

\section{Carbamazepine $\cdot$ Tegretol}

Clonazepam • Klonopin

Donepezil • Aricept

Escitalopram • Lexapro

Gabapentin • Neurontin

\section{Olanzapine • Zyprexa Risperidone $\cdot$ Risperdal Valproate - Depakote Venlafaxine • Effexor}

for teaching them coping skills or strategies to reduce hallucinations, such as distraction, turning on more lights, or even certain eye/blinking movements. ${ }^{11}$ Table $2^{8,11}$ (page 49) outlines behavioral interventions for CBS.

Regardless of etiology, Mr. B's hallucinations significantly affected his quality of life. During his inpatient stay, he was treated with risperidone, $1 \mathrm{mg}$ nightly, because it was determined that the benefits of treatment with an antipsychotic medication outweigh the risks. He was also started on donepezil, $5 \mathrm{mg} / \mathrm{d}$, to treat a possible comorbid dementia. However, his hallucinations continued without significant improvement.

\section{OUTCOME Home care and family involvement}

After discussion with Mr. B and his family about the risks and benefits of medication,

the risperidone and donepezil are discontinued. Ultimately, it is determined that Mr. B requires a higher level of home care, both for his safety and to improve his social contact. Mr. $B$ returns home with a combination of a professional home health aide and increased family involvement.

\section{References}

1. Menon GJ, Rahman I, Menon SJ, et al. Complex visual hallucinations in the visually impaired: the Charles Bonnet syndrome. Surv Ophthalmol. 2003;48(1):58-72.

2. Cox TM, Ffytche DH. Negative outcome Charles Bonnet syndrome. Br J Ophthalmol. 2014;98(9):1236-1239.

3. Pelak VS. Visual release hallucinations (Charles Bonnet syndrome). UpToDate. Updated February 5, 2019. Accessed September 17, 2020. https://www.uptodate. com/contents/visual-release-hallucinations-charlesbonnet-syndrome

4. Burke W. The neural basis of Charles Bonnet hallucinations: a hypothesis. J Neurol Neurosurg Psychiatry. 2002;73(5): 535-541.

5. Scott IU, Schein OD, Feuer WJ, et al. Visual hallucinations in patients with retinal disease. Am J Ophthalmol. 2001;131(5):590-598

6. Lepore FE. Spontaneous visual phenomena with visual loss: 104 patients with lesions of retinal and neural afferent pathways. Neurology. 1990;40(3 Pt 1):444-447.

7. Nesher R, Nesher G, Epstein E, et al. Charles Bonnet syndrome in glaucoma patients with low vision. J Glaucoma. 2001;10(5):396-400.

8. Pang L. Hallucinations experienced by visually impaired Charles Bonnet syndrome. Optom Vis Sci. 2016;93(12):14661478 .

9. Linszen M, Van Zanten G, Teunisse R, et al. Auditory hallucinations in adults with hearing impairment: a large prevalence study. Psychological Medicine. 2019;49(1): 132-139.

10. Teunisse RJ, Cruysberg JR, Hoefnagels WH, et al. Social and psychological characteristics of elderly visually handicapped patients with the Charles Bonnet syndrome. Compr Psychiatry. 1999;40(4):315-319

11. Eperjesi F, Akbarali A. Rehabilitation in Charles Bonnet syndrome: a review of treatment options. Clin Exp Optom. 2004;87(3):149-152.

12. Fernandez A, Lichtshein G, Vieweg WVR. The Charles Bonnet syndrome: a review. J Nen Ment Dis. 1997;185(3): 195-200.

13. Rosenbaum F, Harati Y, Rolak L, et al. Visual hallucinations in sane people: Charles Bonnet syndrome. J Am Geriatr Soc. 1987;35(1):66-68

14. Coletti Moja M, Milano E, Gasverde S, et al. Olanzapine therapy in hallucinatory visions related to Bonnet syndrome. Neurol Sci. 2005;26(3):168-170. 